\title{
The effects of Panax ginseng supplementation on growth hormone, cortisol and lactate response to high-intensity resistance exercise
}

\author{
Leila Zarabi, Hamid Arazi, Mani Izadi \\ Department of Exercise Physiology, Faculty of Sport Sciences, University of Guilan, Rasht, Iran
}

\section{Summary}

Study aim: Growth hormone, cortisol, and lactate play an effective role in regulating and stimulating the muscle building process. The current study aimed to investigate the impact of Panax ginseng supplementation on growth hormone, cortisol, and lactate response to intense resistance exercise in young female non-athletes.

Materials and methods: Ten non-athlete girls participated in this double-blind counter-balanced crossover study. They were assigned to two groups: the ginseng group and the placebo group. In two 4-week periods, they received the supplement and the placebo. In the first four weeks, 5 participants received ginseng (100 mg daily) and 5 others received placebo (maltodextrin). At the end of the 4 weeks, blood samples $(5 \mathrm{ml})$ were taken to measure blood levels of the factors. Afterwards, the resistance exercise protocol was implemented, and immediately after that blood samples were taken. In the second four-week period, the five participants who had taken ginseng received placebo, and the five subjects who had received placebo took ginseng. The same tests were administered again.

Results: The results showed that except for cortisol in the ginseng group, there were significant pre - to post-exercise changes for all 3 indicators in both groups. No significant difference was observed in pre - to post-exercise changes in the levels of GH $(\mathrm{p}=0.71)$, cortisol $(\mathrm{p}=0.34)$, or lactate $(\mathrm{p}=0.90)$ between groups.

Conclusions: $100 \mathrm{mg}$ ginseng consumption daily for 4 weeks does not have an impact on the response of GH, cortisol, or lactate to high-intensity resistance exercise in young female non-athletes.

Keywords: Ginseng - Growth hormone - Cortisol - Lactate - High-intensity resistance exercise

\section{Introduction}

Physical fitness, in particular, the maintenance and development of muscle mass, needs to take advantage of all the sciences, including nutrition and dietary supplements. Therefore, non-athletes are also looking for factors that can even slightly increase their muscle mass. Nowadays, there is a growing tendency to use medical herbs in order to improve body composition and to increase muscle mass, and to improve athletic performance as well [21].

Ginseng is one of the most popular herbal supplementations, and is widely used to enhance physical capacities of both athletes and non-athletes [3]. Ginseng has a high drug property due to the presence of some compounds such as ginsenosides [8]. The pharmacologic effects of ginseng are numerous and multifaceted, and are not only related to the ginsenosides, but are also associated with other compounds such as peptidoglycan, vanillic acid and salicylates, which have antioxidant properties and anti-fatigue effects [19]. The main effects of ginseng are increased physical endurance, improved function of the immune system [5], an adaptogenic effect for increasing muscular mass and strength, elevated resistance against stress and enhanced physical performance [6].

Alterations in the rating of hormonal secretion induced by strength exercise are the main cause of post-exercise protein synthesis and positive adaptation in the structure of skeletal muscle [17]. Growth and cortisol hormones are related to the hypertrophy, and lactate is also considered a factor stimulating anabolic hormones, especially growth hormone $(\mathrm{GH})$. GH is an anabolic hormone secreted from adenohypophysis in response to various stimuli including emotional stresses, fasting, sleep, certain amino acids, some drugs and exercise [31]. Anabolic effects of GH occur in almost all tissues [4]. Many previous studies indicated that severe resistance exercise stimulates human $\mathrm{GH}$ secretion $[9,25]$. 
Conversely, cortisol is known as a catabolic hormone and is released from the adrenal gland and some other tissues such as adipose, liver and muscle, playing a role in breakdown of proteins, triglyceride and fatty acids [18]. The secretion of cortisol is performed by stimulating adrenocorticotropic hormone (ACTH), and it is regulated by the mechanism of hypothalamus-pituitary feedback. It is demonstrated that intense exercise causes elevated plasma cortisol in males and the response depends on the magnitude of exercise stress [10, 23, 25].

Lactate is one of the final products of anaerobic metabolism which is formed from glucose in the absence of oxygen. Accumulated lactate disturbs muscular contraction and results in fatigue [33]. Some studies reported decreased lactate production as the result of ginseng intake $[21,26,27]$ and some others [29] showed no change.

Erhrnborg et al. [12] and Hansen et al. [20] reported a strong relationship between the level of blood lactate and GH secretion. Engels and Wirth [13] indicated that 8 -week ginseng consumption resulted in no significant improvement in the performance of healthy men within submaximal and maximal exercise. Similarly, another investigation on the use of $3 \mathrm{~g}$ ginseng supplement for 8 weeks found no significant effect on lactate threshold and performance among young men [29]. Kang et al. [22] reported that taking $15 \mathrm{~g}$ of ginseng root extract after intense resistance exercise in men did not cause a significant change in the response of $\mathrm{GH}$, testosterone, cortisol or IGF-1.

In contrast, Hsu et al. [21] revealed a significant decrease in plasma creatine kinase and lactate after 4 weeks of ginseng use ( $400 \mathrm{mg}$ per day) in men. However, aerobic work capacity remained unchanged. Likewise, 2-week use of ginseng (4 capsules of $250 \mathrm{mg}$ per day) in conjunction with resistance training resulted in significant enhancement in bench press and squat strength [1]. McNaughton et al. [27] also reported an increase in the strength of pectoralis and quadriceps muscles alongside improved post-exercise recovery induced by ginseng use.

Taking account of the literature, it seems that most of the studies have investigated the effect of ginseng supplementation on aerobic performance or muscular strength, and it is not very clear how ginseng affects strength. Nevertheless, the use of ginseng not only accelerates recovery from fatigue or injuries, but also may affect up-regulation of the hormonal response to anaerobic exercise. This effectiveness would partly be attributed to possible alterations in anabolic and catabolic hormones, and lactate as a factor stimulating anabolic hormones, especially GH. Thus, the present research was conducted to determine the effect of 4-week ginseng supplementation on the GH, cortisol and lactate changes after intense resistance exercise in non-athlete girls.

\section{Materials and methods}

\section{Participants}

The present double-blind semi-experimental study was conducted in the form of placebo-controlled crossover design. Among young female non-athlete students (Guilan University) aged 20 to 24,10 subjects were selected. Inclusion criteria were as follows: lack of cardio-respiratory diseases; no use of tobacco, drugs or supplements; regular menstrual cycle; free of any restriction on doing exercise; and lack of musculoskeletal injury. Demographic characteristics of the subjects are presented in Table 1. A preliminary session was held with the aim of familiarization with the process including study aim, various stages of the research, how to perform exercises, the manner of supplement usage, and being aware of possible injuries caused by exercises.

\section{Study protocol}

Subjects were randomly assigned to two groups of five members, and they used ginseng and placebo during two 4-week periods. Ginseng supplement (G115 Panax ginseng C.A. Meyer, Ginsana SA Company, Switzerland) and maltodextrin were provided in $100 \mathrm{mg}$ capsules for experimental and placebo groups, respectively. Two groups used provided capsules for 4 weeks. That is, in the first 4 weeks, half of the participants consumed ginseng and the others used placebo, and vice versa for the next 4 weeks. At the end of each 4-week period, a high-intensity resistance exercise protocol along with blood sampling (immediately after exercise) was accomplished. It is noteworthy that subjects were instructed to follow their regular diet and physical activity within two 4-week periods. Subjects were also asked to avoid consuming alcohol and caffeine, and were invited to take part in the testing procedure after

Table 1. Demographic characteristics of the subjects

\begin{tabular}{lc}
\hline Variables & $\mathrm{M} \pm \mathrm{SD}$ \\
\hline Age $[\mathrm{yr}]$ & $23.4 \pm 0.69$ \\
Height $[\mathrm{cm}]$ & $163 \pm 1.76$ \\
Weight $[\mathrm{kg}]$ & $57.61 \pm 6.9$ \\
$\mathrm{BMI}\left[\mathrm{kg} \cdot \mathrm{m}^{-2}\right]$ & $21.76 \pm 2.81$ \\
\hline $1 \mathrm{RM}[\mathrm{kg}]$ & \\
\hline Leg press & $112.8 \pm 24.18$ \\
Lat pull-down & $28.65 \pm 3.7$ \\
Leg curl & $22.35 \pm 5.7$ \\
Bench press & $22.35 \pm 7.15$ \\
Leg extension & $17.7 \pm 3.15$ \\
Barbell overhead press & $16.25 \pm 3.7$ \\
\hline
\end{tabular}


$36 \mathrm{~h}$ resting and $12 \mathrm{~h}$ fasting. The procedures were approved by the Institutional Ethics Review Committee of the University of Guilan.

\section{RM assessment}

One-repetition maximum (1RM) strength of the subjects for 6 exercises - leg press, lat pull-down, leg curl, bench press, leg extension, and barbell overhead press was determined using the Brzycki equation [7]. That is, after 15 minutes of warm-up, each of 6 exercises was performed with a load almost equal to the maximum for 8 repetitions. The numbers of repetitions plus the magnitude of the load then were used to estimate 1RM.

$$
1 \mathrm{RM}=100 \times \text { load } \mathrm{rep} /(102.78-2.78 \times \mathrm{rep})
$$

\section{Resistance exercise protocol}

Prior to the exercise session, 10 minutes were devoted to warm-up consisting of jogging, stretching and dynamic movements. Afterwards, 6 resistance exercises with $80 \%$ of $1 \mathrm{RM}$ were performed in 25 minutes. Three sets of 8 to 10 repetitions were considered for exercises with 1 - and 2-minute rest intervals between sets and exercises, respectively. At the end of an exercise session, 10 minutes were also spent doing cool-down including static stretching.

\section{Blood sampling}

Prior to the blood sampling (7:00 am), a stress questionnaire was filled out by subjects with the aim of controlling the stress of participants. Approximately $5 \mathrm{ml}$ blood as the pre-test was collected from the brachial vein before the exercise protocol. Immediately after the exercise session, a secondary blood sampling was done as the post-test. In order to minimize the effects of circadian rhythm and the menstrual cycle, the blood samples were taken at the same time of day and in the luteal phase. Blood samples transferred to the laboratory immediately after collection, were encoded, centrifuged and kept at $-70^{\circ} \mathrm{C}$. Eventually, blood samples were assessed in order to determine lactate levels (Enzymatic calorimetry, Lactate Mono Liquid kit, BIOREX UK, with the sensitivity of $2 \mathrm{mg} / \mathrm{dl}$ ), cortisol (Enzyme immunoassays (EIA), GmbH kit, made in Germany, with a coefficient of variation of less than 9\%) and GH (ELISA, Monobind, made in USA).

\section{Statistical analyses}

After testing normality of data using the KolmogorovSmirnov test, the paired t-test and independent t-test were used to determine intragroup changes and intergroup differences in the percentage of changes, respectively. All the statistical analyses were performed using SPSS 22 and Excel 2010. The significance level was set at $\mathrm{P}<0.05$.

\section{Results}

The findings of the present study showed that after 4 weeks of ginseng supplementation, both groups showed a significant increase $(\mathrm{P}=0.03)$ in $\mathrm{GH}$ subsequent to highintensity resistance exercise. There was no significant difference $(\mathrm{P}=0.71)$ between groups in $\mathrm{GH}$ (Fig. 1).

Both groups demonstrated an elevated post-exercise cortisol level, but this increase was significant $(\mathrm{P}=0.001)$ only for the placebo group, and the ginseng group showed a non-significant $(\mathrm{P}=0.61)$ increase. Further, the difference between the two groups in terms of the percentage of changes in cortisol was not significant ( $\mathrm{P}=0.34$ ) (Fig. 2).

Similar to the results of $\mathrm{GH}$, lactate had a significant $(\mathrm{P}=0.001)$ post-exercise increase in both groups. Moreover, a non-significant difference $(\mathrm{P}=0.90)$ pertaining to the percentage of pre- to post-exercise changes in lactate was found between the two groups (Fig. 3).

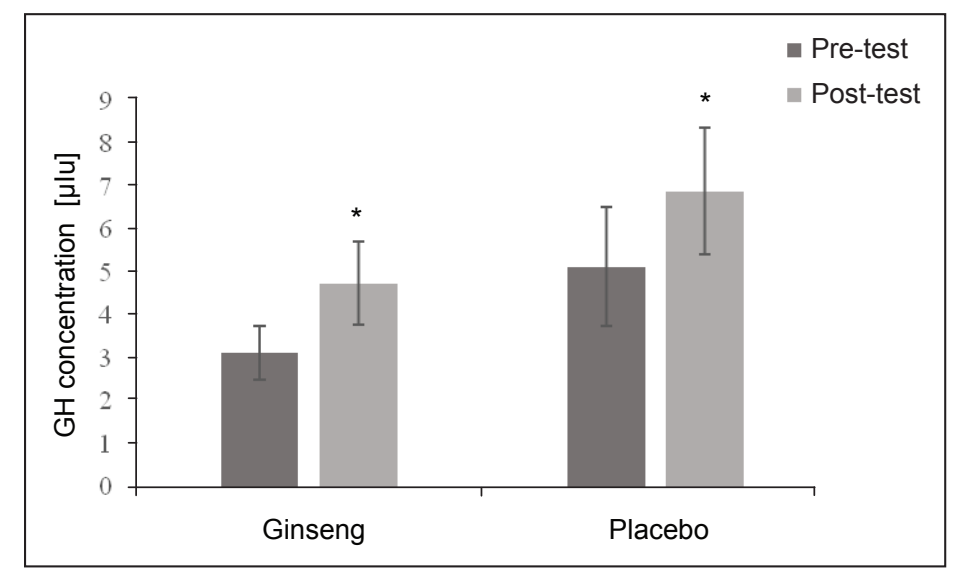

Fig. 1. Pre- to post-exercise changes in GH levels for ginseng and placebo groups. * Significantly different in comparison with pre-test. 


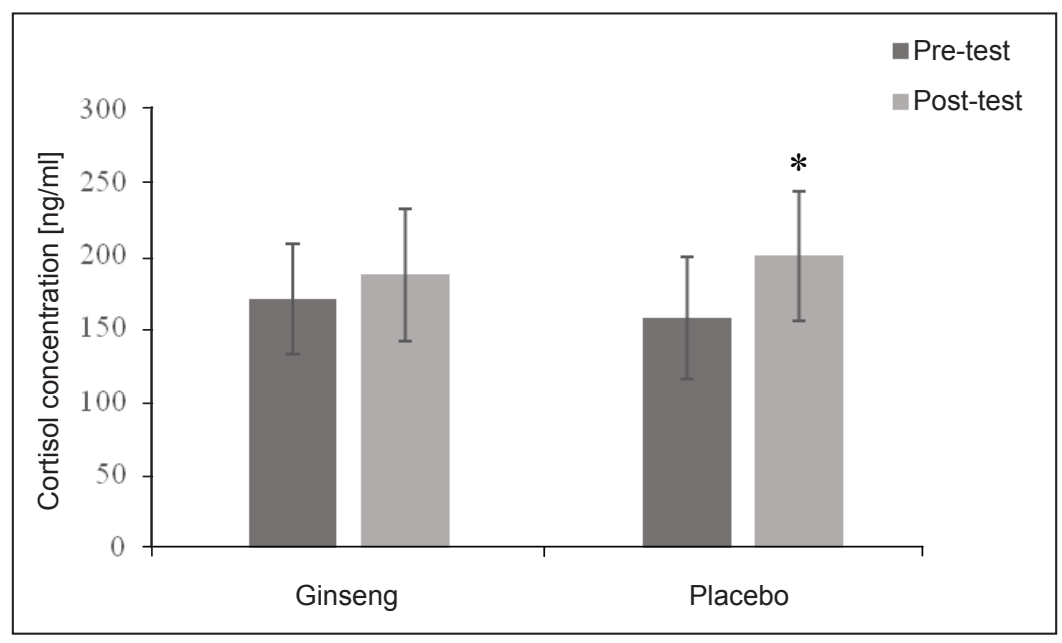

Figure 2. Pre- to post-exercise changes in cortisol levels for ginseng and placebo groups. * Significantly different in comparison with pre-test

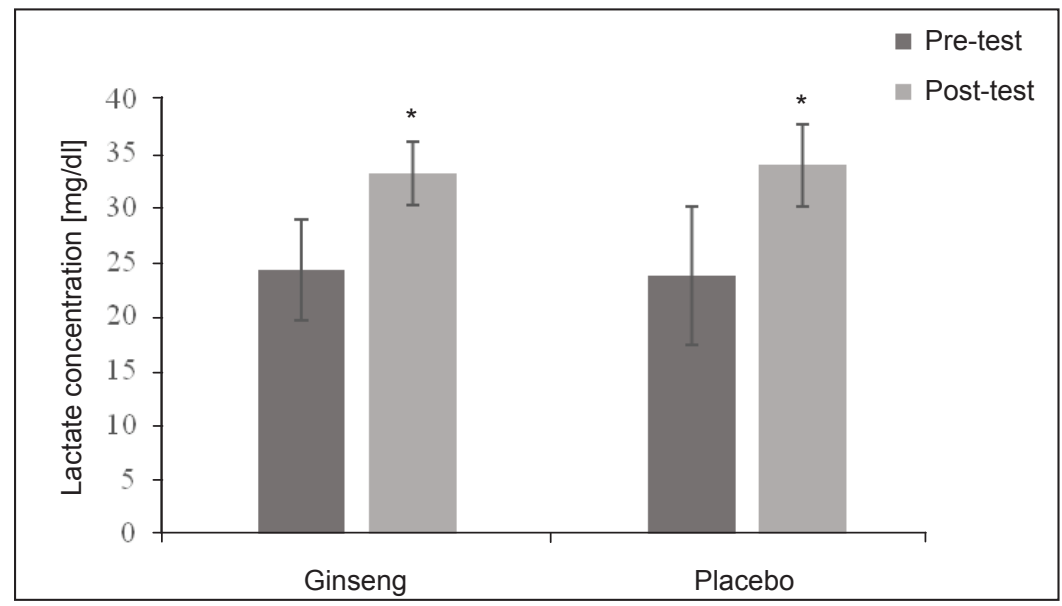

Fig. 3. Pre- to post-exercise changes in lactate levels for ginseng and placebo groups. * Significantly different in comparison with pre-test

\section{Discussion}

High-intensity resistance exercise causes further protein synthesis and increased muscular strength. In addition to being influenced by the volume and intensity of exercise, this increase in strength is also affected by the hormonal environment of the active muscles. Growth and cortisol hormones and blood lactate are factors contributing to anabolic responses to resistance exercise. According to the literature, ginseng supplementation results in improved bioenergy production and muscular strength $[2,32]$, suggesting an effect on protein metabolism through stimulated anabolic pathways. Hence, this research sought to elucidate the effect of 4 weeks of ginseng supplementation on $\mathrm{GH}$, cortisol and lactate response to high-intensity resistance exercise among young female non-athletes.

The results showed that 4 weeks of ginseng supplementation, $100 \mathrm{mg}$ per day, has no significant effect on $\mathrm{GH}$, cortisol and lactate response to high-intensity resistance exercise. The findings are consistent with the results of Kang et al. [22], Morris et al. [28] and Kulaputana et al. [29], and ginseng ingestion did not exert an ergogenic effect. Kang et al. [22] reported no significant alteration of $\mathrm{GH}$, testosterone, cortisol or IGF-1 response to acute use of $15 \mathrm{~g}$ of ginseng root extract after intense resistance exercise among men.

The finding of the present research pertaining to $\mathrm{GH}$ was similar to preceding studies. Some potential mediators for non-significant changes would be the low dosage of ginseng, short period of supplementation, and the lack 
of repeated sampling several times after exercise. Godfrey et al. [16] indicated that the secretion of GH is greatly increased after resistance exercise, which can partly be attributed to the accumulation of lactate. In the same study, the increase in lactate was not significant, and this can explain the non-significant elevation of GH.

In the present research, cortisol showed a significant increase in the placebo group, while in the ginseng group the increase in cortisol was non-significant. However, no significant difference was found between the two groups. The mechanism involved in the secretion of cortisol can be mediated by corticotropin-releasing hormone (CRH), a peptide hormone stimulating both the synthesis and the secretion of adrenocorticotropic hormone (ACTH) from adenohypophysis, resulting in the secretion of cortisol from adrenal glands [11].

Ginseng also affects CNS, and ginsenosides mainly exert their impact through the hypothalamic-pituitaryadrenal axis (HPA axis) [34]. Although ginseng ingestion showed such an anti-stress effect and partly adjusted the post-exercise increment of cortisol, there was no significant difference between the two groups in terms of pre - to post-exercise alterations in the cortisol levels.

Kraemer et al. [24] reported an elevation in creatine kinase (CK), which is a sign of muscle injury, after resistance exercise. Given that the anti-inflammatory effect of cortisol is well known, its secretion can rise in response to muscle injury induced by resistance exercise. In this context, in a study 13 male students were divided into two groups of ginseng and placebo, and trained with $80 \%$ of $\mathrm{VO}_{2 \max }$ for 4 weeks. Eventually, $\mathrm{CK}$ and lactate dropped in the ginseng group compared to the placebo group [21]. In the same research, ginseng reduced $\mathrm{CK}$, suggesting diminished muscle injury. Gaffney et al. [15] also conducted a 6-week study into the impacts of ginseng on exercisegenerated stress and some hormonal responses among male athletes. The findings showed no influence on cortisol and testosterone.

In another study, Gaffney et al. [14] investigated the contradictory effects of ginseng, which sometimes suppresses and even sometimes stimulates physiological stress. The results showed that ginseng inhibits 11-beta hydroxysteroid dehydrogenase one, which is adjacent to the receptors of stress hormones, has a destructive impact on them, and makes them inactive. The authors concluded that the enhanced energy resulted from ginseng intake may also be due to the increased stress hormone receptors.

Based on another finding of the present research, pre to post-exercise lactate changes were significant for both groups. This increment was greater for the placebo group, indicating a minimal capacity of ginseng for adjusting lactate concentration. However, no significant difference was observed between groups. These results are in contrast to some previous findings suggesting decreased lactate [21,
26, 27] and are consistent with some others [29] demonstrating a lack of change in lactate as a result of ginseng ingestion.

Wong et al. [35] reported that acute supplementation of $200 \mathrm{mg}$ of Panax ginseng reduces plasma lactate concentration. In contrast, Morris et al. [28] and Ping et al. [30] revealed no ergogenic property of ginseng in reducing post-exercise lactate concentration. The findings of the present research confirm the results reported in two previous studies, suggesting no adaptogenic effect of ginseng in terms of adjusting post-exercise lactate concentration. However, it is noteworthy that previous studies mainly investigated whether or not these effects are observed after endurance exercises.

\section{Conclusion}

Collectively, based on the findings of the present study, it can be concluded that 4-week supplementation of 100 mg ginseng has no effect on hormonal responses following high-intensity resistance exercise. However, these results can be explained by the small numbers of measurements (just pre-exercise and immediately after exercise) and limited subjects. Furthermore, the type and the dosage of ingested ginseng, plus non-athlete participants, may also contribute to these findings.

The same study is suggested to be conducted on athletes and non-athletes with the purpose of comparison of the adaptogenic effects of ginseng among them. Moreover, in this research only single session resistance exercise was considered. Thus, investigating long-term hormonal adaptations of resistance training alongside ginseng supplementation with the same dosage as used in the present research appears to be valuable in order to elucidate further data more precisely.

Conflict of interest: Authors state no conflict of interest.

\section{References}

1. Arzeghar M., A. Hematfar, S.M. Naghibzadeh, J. Rashnavadinejad (2014) Effect of panax ginseng on indicators of physiological, blood biochemical, and body composition during strength training of male power lifting athletes. IJSS, 4(4): 380-385.

2. Bahrke M.S., W.P. Morgan (1994) Evaluation of the ergogenic properties of ginseng. Sports Med., 18(4): 229-248.

3. Bahrke M.S., W.P. Morgan, A. Stegner (2009) Is ginseng an ergogenic aid? Int. J. Sport Nutr. Exerc. Metab., 19(3): 298-322. 
4. Borst S.E., D.V. De Hoyos, L. Garzarella, K. Vincent, B.H. Pollock, D.T. Lowenthal, M.L. Pollock (2001) Effects of resistance training on insulin-like growth factor-I and IGF binding proteins. Med. Sci. Sports Exerc., 33(4): 648-653.

5. Brekhmam I., I. Dardymov (1969) Pharmacological investigations of glycosides from ginseng and Eleutherococcus. Lloydia, 32: 46-51.

6. Briskin D.P. (2000) Medicinal plants and phytomedicines. Linking plant biochemistry and physiology to human health. Plant Physiol., 124(2): 507-514.

7. Brzycki M. (1993) Strength testing - predicting a one-rep max from reps-to-fatigue. J. Phys. Educ. Recreat. Dance, 64(1): 88-90.

8. Chong S., V. Oberholzer (1988) Ginseng--is there a use in clinical medicine? Postgrad. Med. J., 64(757): 841-846.

9. Craig B.W., H.Y. Kang (1994) Growth Hormone Release Following Single Versus Multiple Sets of Back Squats: Total Work Versus Power. J. Strength Cond. Res., 8(4): 270-275.

10. Cumming D.C., L. Brunsting 3rd, G. Strich, A.L. Ries, R.W. Rebar (1986) Reproductive hormone increases in response to acute exercise in men. Med. Sci. Sports Exerc., 18(4): 369-373.

11. Durand R.J., V.D. Castracane, D.B. Hollander, J.L. Tryniecki, M.M. Bamman, S. O’Neal, E.P. Hebert, R.R. Kraemer (2003) Hormonal responses from concentric and eccentric muscle contractions. Med. Sci. Sports Exerc., 35(6): 937-943.

12. Ehrnborg C., K. Lange, R. Dall, J. Christiansen, P.-A. Lundberg, R. Baxter, M.A. Boroujerdi, B.A. Bengtsson, M.L. Healey, C. Pentecost, S. Longobardi, R. Napoli, T. Rosén (2003) The growth hormone/insulinlike growth factor-I axis hormones and bone markers in elite athletes i $\mathrm{n}$ response to a maximum exercise test. J. Clin. Endocrinol. Metab., 88(1): 394-401.

13. Engels H.-J., J.C. Wirth (1997) No ergogenic effects of ginseng (Panax ginseng CA Meyer) during graded maximal aerobic exercise. J. Am. Diet. Assoc., 97(10): 1110-1115.

14. Gaffney B., H. Hügel, P. Rich (2001) Panax ginseng and Eleutherococcus senticosus may exaggerate an already existing biphasic response to stress via inhibition of enzymes which limit the binding of stress hormones to their receptors. Med. Hypotheses, 56(5): 567-572.

15. Gaffney B.T., H.M. Hügel, P.A. Rich (2001) The effects of Eleutherococcus senticosus and Panax ginseng on steroidal hormone indices of stress and lymphocyte subset numbers in endurance athletes. Life Sci., 70(4): 431-442.

16. Godfrey R.J., Z. Madgwick, G.P. Whyte (2003) The exercise-induced growth hormone response in athletes. Sports Med., 33(8): 599-613.

17. Goto K., N. Ishii, K. Takamatsu (2004) Growth hormone response to training regimen with combined high-and low-intensity resistance exercises. Int. J. Sport Health Sci., 2: 111-118.

18. Goulet E.D., A. Hassaine, I.J. Dionne, P. Gaudreau, A. Khalil, T. Fulop, B. Shatenstein, D. Tessier, J.A. Morais (2009) Frailty in the elderly is associated with insulin resistance of glucose metabolism in the postabsorptive state only in the presence of increased abdominal fat. Exp. Geront., 44(11): 740-744.

19. Hallstrom C., S. Fulder, M. Carruthers (1978) Effects of ginseng on the performance of nurses on night duty. Am. J. Chin. Med., 6(04): 277-282.

20. Hansen S., T. Kvorning, M. Kjaer, G. Sjøgaard (2001) The effect of short-term strength training on human skeletal muscle: the importance of physiologically elevated hormone levels. Scand. J. Med. Sci. Sports, 11(6): 347-354.

21. Hsu C.-C., M.-C. Ho, L.-C. Lin, B. Su, M.-C. Hsu (2005) American ginseng supplementation attenuates creatine kinase level induced by submaximal exercise in human beings. World J. Gastroenterol., 11(34): 5327.

22. Kang H.Y., S.H. Kim, W.J. Lee, H.K. Byrne (2002) Effects of ginseng ingestion on growth hormone, testosterone, cortisol, and insulin-like growth factor 1 responses to acute resistance exercise. J. Strength Cond. Res., 16(2): 179-183.

23. Kraemer W.J., S.E. Gordon, S.J. Fleck, L. Marchitelli, R. Mello, J.E. Dziados, K. Friedl, E. Harman, C. Maresh, A.C. Fry (1991) Endogenous anabolic hormonal and growth factor responses to heavy resistance exercise in males and females. Int. J. Sports Med., 12(02): 228-235.

24. Kraemer W.J., K. Häkkinen, R.U. Newton, B.C. Nindl, J.S. Volek, M. McCormick, L.A. Gotshalk, S.E. Gordon, S.J. Fleck, W.W. Campbell, M. Putukian, W.J. Evans (1999) Effects of heavy-resistance training on hormonal response patterns in younger vs. older men. J. Appl. Physiol., 87(3): 982-992.

25. Kraemer W.J., L. Marchitelli, S.E. Gordon, E. Harman, J.E. Dziados, R. Mello, P. Frykman, D. McCurry, S.J. Fleck (1990) Hormonal and growth factor responses to heavy resistance exercise protocols. J. Appl. Physiol., 69(4): 1442-1450.

26. Lee N.-H., C.-G. Son (2011) Systematic review of randomized controlled trials evaluating the efficacy and safety of ginseng. J. Acupunct. Meridian Stud., 4(2): 85-97.

27. McNaughton L., G. Egan, G. Caelli (1989) A comparison of Chinese and Russian ginseng as ergogenic aids to improve various facets of physical fitness. Int. Clin. Nutr. Rev., 9(1): 32-35.

28. Morris A.C., I. Jacobs, T.M. McLellan, A. Klugerman, L.C. Wang, J. Zamecnik (1996) No ergogenic effect of ginseng ingestion. Int. J. Sport Nutr., 6(3): 263-271.

29. Onanong Kulaputana M. (2007) Ginseng supplementation does not change lactate threshold and physical 
performances in physically active Thai men. J. Med. Assoc. Thai., 90(6): 1172-1179.

30. Ping F.W.C., C.C. Keong, A. Bandyopadhyay (2011) Effects of acute supplementation of Panax ginseng on endurance running in a hot \& humid environment. Indian $J$. Med. Res., 133(1): 96.

31. Stone M.H. (1995) Literature review: Human Growth Hormone: Physiological Functions and Ergogenic Efficacy. Strength Cond. J., 17(4): 72-74.

32. Takamura Y., Y. Makanae, S. Ato, N. Yoshii, K. Kido, M. Nomura, A. Uchiyama, N. Shiozawa, S. Fujita (2016) Panaxatriol derived from ginseng augments resistance exercised-induced protein synthesis via mTORC1 signaling in rat skeletal muscle. Nutr. Res., 36(11): 1193-1201.

33. Thompson P.D., B.A. Franklin, G.J. Balady, S.N. Blair, D. Corrado, N.M. Estes, J.E. Fulton, N.F. Gordon, W.L. Haskell, M.S. Link, B.J. Maron, M.A. Mittleman, A. Pelliccia, N.K. Wenger, S.N. Willich, F. Costa (2007)
Exercise and acute cardiovascular events. Circulation, 115(17): 2358-2368.

34. Vogler B., M. Pittler, E. Ernst (1999) The efficacy of ginseng. A systematic review of randomised clinical trials. Eur. J. Clin. Pharmacol., 55(8): 567-575.

35. Wong C.P.F., A. Bandyopadhyay, C.K. Chen (2011) Effects of Panax ginseng supplementation on physiology responses during endurance performance. J. Mens Health, 8: S78-S80.

\section{Received 23.10.2017 \\ Accepted 02.01.2018}

(C) University of Physical Education, Warsaw, Poland

Acknowledgments

The authors would like to acknowledge all the subjects who volunteered to participate in this research. 